\title{
Increased Anion Gap Metabolic Acidosis as a Result of 5- Oxoproline (Pyroglutamic Acid) in Pregnancy: A Case Report
}

\author{
Sathiyathasan S, Jeyanthan K, Soper H, Hamid R \\ Mayday University Hospital, Croydon, Surrey UK
}

\begin{abstract}
Accumulation of 5- Oxoproline (pyroglutamic acid) is a rare cause of severe, high anion gap metabolic acidosis in adults. A 21 year old lady presented at 39 weeks gestation in her first pregnancy with 2 weeks history of shortness of breath. Arterial blood gas showed metabolic acidosis. Urine and serum samples were taken which showed an increase in the serum level of 5- Oxoproline -creatinine ratio indicating pyroglutamic metabolic acidosis. Metabolic acidosis that is caused by 5- oxoproline results from disruption of the gamma glutamyl cycle. Glutamile synthetase (GS) deficiency is an autosomol recessive disorder. With GS deficiency, reduced glutathione levels increases gamma glutamile synthetase activity, and the resulting gamma - glutamile cystine levels are converted to 5-oxoproline.Suspicion for 5-oxoproline-associated high anion gap metabolic acidosis should be made when the cause of high anion gap metabolic acidosis remains poorly defined.
\end{abstract}

Keywords: Acetaminophen, anion gap, metabolic acidosis, polyglutamic aciduria, pregnancy

\section{INTRODUCTION}

A rare cause of high anion gap acidosis is 5-oxoproline (pyroglutamic acid), an organic acid intermediate of the $\gamma$-glutamyl cycle. Polyglutamic acidemia should be considered when most common causes such as ketoacidosis, lactic acidosis or ingestion of salicylates, methanol or ethynyl glycol are excluded. ${ }^{1}$

\section{CASE}

A 21 year old lady presented at 39 weeks gestation in her first pregnancy with 2 weeks history of shortness of breath. She suffered from earache and had been taking paracetamol on regular basis for a year. She was admitted twice during the course of pregnancy. First time she was treated for urinary tract infection with amoxicillin and then treated for chest infection with erythromycin. She admitted to having regular alcohol intake until the pregnancy when she stopped. There was no history of diabetes, hypertension or renal disease.

Her respiratory rate and blood pressure were normal but her pulse rate was $105 \mathrm{bpm}$.The baseline laboratory analysis revealed her hemoglobin to be $10.3 \mathrm{~g} / \mathrm{dl}$ with high MCV. Arterial blood gas showed metabolic acidosis (table 1). Her white cell count was 20,500/ul. Blood urea, creatinine and electrolytes all were within normal limits at that time. Liver enzymes showed increased gamma glutamyl transfer rates to the level of $202 \mathrm{u} / \mathrm{l}$. The anion gap $34 \mathrm{mEq} / \mathrm{l}$ was consistent with metabolic acidosis. (The normal range should be less than $13 \mathrm{mEq} / \mathrm{l}$ ). Plasma amino acid screen revealed no abnormality.

\section{CORRESPONDENCE}

Dr S Sathiyathasan, MRCOG

SPR, Department of Obstetrics and Gynecology

Mayday University Hospital, Croydon Surrey UK

Phone: 00447904345476

Email: sathya@doctors.org.uk 
Table 1. Blood Gas

\begin{tabular}{lc}
\hline & Blood Gas \\
\hline $\mathrm{PH}$ & 7.26 \\
$\mathrm{Pco} 2$ & 1.05 \\
$\mathrm{Po} 2$ & 26.0 \\
$\mathrm{HCO} 3-$ & 8.9 \\
Base Excess & -23.6 \\
Lactate & 1.6
\end{tabular}

Plasma $\gamma$ glutamyl transferase level was 202u/l (6-42). Chest X-rays showed patchy consolidation keeping with a chest infection. From the time of admission, she was put on continuous fetal heart cardiotocographic (CTG) monitoring. At this stage, the CTG showed unprovoked decelerations and emergency cesarean section was carried out. Urine and serum samples were taken which showed an increase in the serum level 5- Oxoproline - Creatinine ratio indicating pyroglutamic metabolic acidosis. Polyglutamate was found in the urine.

As she had no renal impairment differential diagnosis made was intoxication with organic acids but there was no evidence of ethanol, methanol or ethylene glycol ingestion. She was admitted to intensive care unit. She underwent ventilation and hemofiltration. On the second day of admission metabolic acidosis was corrected. During the 48 hours treatment with supportive therapy, overall improvement was seen.

\section{COMMENT}

Pyroglutamic metabolic acidosis is a condition which can be caused by pregnancy, anorexia, alcohol abuse, chronic paracetamol ingestion, liver or renal disease. Polyglutamic academia (5-oxoprolinemia) occurs as a result of overproduction of polyglutamic acid secondary to deficiency of glutathione synthetase (GS) of failure of breakdown of polyglutamic acid by 5-oxoprolinase 1 .

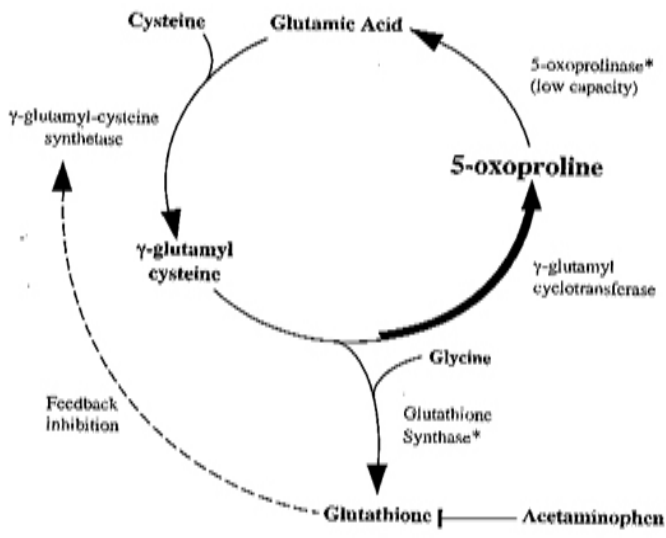

Figure 1. The X-glutamyl cycle: 5 -oxoproline is an organic acid intermediate, Acetaminophen reduces intracellular glutathione levels
Several inherited enzyme defects that have been characterised are extremely rare and usually presents at a very early age with neurologic and hematologic abnormalities. GS deficiency is an autosomol recessive disorder. With GS deficiency, reduced glutathione levels increase $\gamma$ glutamile synthetase activity, and the resulting $\checkmark$ glutamile cystine levels are particularly converted to 5-oxoproline (Fig 1)

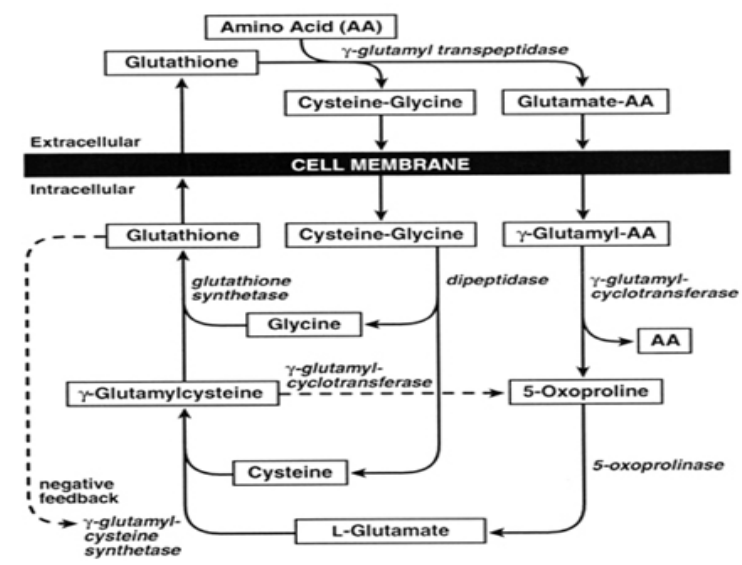

Figure 2. Gamma-glutamyl cycle

We believe our patient may have had heterozygosity for glutathione synthetase deficiency. Heterozygote state is characterised by a moderate reduction (50\%) in the activity of glutathione synthetase, normal baseline glutathione levels and no clinical abnormality. Acquired 5- Oxoprolinuria has been described in adults. Several contributory factors include malnutrition, pregnancy and strict vegetarian diet. $^{2}$ Severe 5 - oxoproline aciduria and academia has been described in patients who use several different medications that include acetaminophen (paracetamol), vigabatrin and the antibiotics flucloxacillin and netilmicin. ${ }^{3}$

High anion gap metabolic acidosis occurs frequently in patients with acetaminophen toxicity. This generally is attributed to lactic acidosis and kidney failure. However in some cases anion gap cannot be explained. These patients often have less severe toxicity and history of chronic acetaminophen ingestion. ${ }^{4}$

In a literature review 18 adult cases of 5 - Oxoprolinuria and high anion gap metabolic acidosis associated with chronic therapeutic ingestion of acetaminophen, 14 out of 18 described patients were women. The activity of several isoenzymes in the $\gamma$ glutamyl cycle is known to be different in men and women. These differences may increase the susceptibility to women to develop this disorder. ${ }^{5}$ Enhanced toxicity of acetaminophen abusers of alcohol and the drugs can be expected for several reasons: Firstly, in human studies, acute alcohol intake has been shown to decrease toxic metabolic activation (mixed function oxidase system) of paracetamol in liver 
micrososmes. ${ }^{7}$ Serious hepatotoxicity at recommended or near recommended doses for therapeutic purposes has been reported in association with chronic alcohol use as in chronic alcoholics as they predisposes to paracetamolrelated toxicity at relatively low doses. ${ }^{8}$ This again was a possible contributing factor in this case.

Secondly, poor diet in the alcoholic may enhance the toxicity of acetaminophen. Thirdly, preexistent liver disease may increase the risk of toxic liver injury. Finally, the alcoholics may be more likely than the general public to exceed the recommended doses of over the counter drugs. ${ }^{9}$

Polyglutamic acidemia may be seen in association with pregnancy when glycine availability is limited. Generally, the levels of polyglutamate are much less marked in these conditions than those described in the inherited disorders of metabolism. Metabolic acidosis could lead to fetal acidosis and electrolyte imbalance. ${ }^{10}$

Also studies showed increasing metabolic acidosis was accompanied by decreasing rate of blood flow in the uterine circulation. ${ }^{11}$ Sepsis or sepsis like systemic inflammatory response can be associated with pyroglutamic acidemia which is explained by animal studies. Liver glutathione can be depressed in established septic shock. This will reduce the feedback inhibition of $\gamma$ glutamyl cysteine synthetase, allowing the increased production of pyroglutamic acid and cysteine. ${ }^{12}$

These causes could have contributed to fetal distress as in this case. Management of severe polyglutamic acidemia involves supportive measures aimed at preservation and optimization of respiratory, cardiovascular and renal function. The presence of infection should be sought and treated accordingly.

\section{CONCLUSIONS}

Acetaminophen, a popular nonprescription analgesic and antipyretic drug, is available in various dosage levels as a generic compound as well as in combination with other agents. The chronic use of over the counter medication could potentially be hazardous in certain patient populations. For example the long term use of acetaminophen even at therapeutic doses can lead to complications in chronic alcoholics. Moreover this patient could have been heterozygote for glutathione synthetase deficiency which increased the risk for toxicity. Obtaining detailed drug history and personal history is of prime importance to assess the risk even with acetaminophen which is a commonly used drug in the absence of other metabolic causes.

Suspicion for 5-oxoproline-associated high anion gap metabolic acidosis should be made when the cause of high anion gap metabolic acidosis remains poorly defined, the anion gap cannot be explained reasonably by measured organic acids, and there is concomitant acetaminophen use. Clinicians need to be aware of this unusual cause of anion gap acidosis because it may be more common than expected. Early discontinuation of the offending agent is therapeutic and administration of $\mathrm{N}$-acetylcysteine could be beneficial.

\section{REFERENCES}

1. Dempsey GA, Lyall HJ, Corke CF, Scheinkestel CD. Pyroglutamic acidemia: a cause of high anion gap metabolic acidosis. Crit Care Med. 2000;28(6):1803-7

2. Fenves AZ, Kirkpatrick HM 3rd, Patel VV, Sweetman L, Emmett M. Increased anion gap metabolic acidosis as a result of 5-oxopronoline (pyroglutamic acid): a role for acetaminophen. Clic J Am Soc Nephrol. 2006;1(3): 441-7.

3. Pitt J. Association between paracetamol and pyroglutamic aciduria. Clin Chem. 1990;36(1):173-4.

4. Record CO, Iles RA, Cohen RD, Williams R. Acid-base and metabolic disturbances in fulminant hepatic failure. Gut.1975;16(2):144-9.

5. Butera L, Feinfeld DA, Bhargava M. Sex differences in the subunits of glutathione-S-transferase isoenzyme from rat and human kidney. Enzyme.1990;43(4):175-182

6. Spielberg SP. In vitro assessment of pharmacogenetic susceptibility to toxic drug metabolites in humans. Fed Proc. 1984;43(8):2308-13.

7. Thummel KE, Slattery JT, Nelson SD, Lee CA, Pearson PG. Effect of ethanol on hepatotoxicity of acetaminophen in mice and on reactive metabolite formation by mouse and human liver micrososmes. Toxicol Appl Pharmacol. 1989;100(3):391-7.

8. Riordan SM, Williams R. Alcohol exposure and paracetamol-induced hepatotoxicity. Addict Biol. 2002;7(2):191-206.

9. Leist MH, Gluskin LE, Payne JA. Enhanced toxicity of acetaminophen in alcoholics : report of three cases. J Clin Gastroenterol.1985;7(1):55-9.

10. Kamalakannan D, Baskar V, Barton DM, Abdu TA. Diabetic ketoacidosis in pregnancy. Postgrad Med J. 2003;79(934):454-7.

11. Blechner JN, Stenger VG, Prystowsky H. Blood flow to the human uterus during maternal metabolic acidosis. Am J Obstet Gynaecol. 1975;121(6):789-94

12. Colomb V, Petit J, Matheix-Fortunet H, Hecketsweiler B, Kaeffer $\mathrm{N}$, Lerebours $\mathrm{E}$, et al. Influence of antibiotics and food intake on liver glutathione and cytochrome $\mathrm{P}-450$ in septic rats. Br J Nutr 1995;73(1):99-110. 